\title{
Una aproximación a Hendrik Weyenbergh y Manuel L. Lucero, el primer Decano de la Facultad de Ciencias Médicas y el Rector de la Universidad Nacional de Córdoba en el marco de sus circunstancias histórico- sociales
}

\author{
An approach to Hendrik Weyenbergh and Manuel L. Lucero, the first Dean of the Faculty of Medical Sciences and the \\ Rector of the National University of Córdoba within the framework of their social-historical circumstances
}

\section{Aldo R. Eynard'}

1. Profesor Emérito de la Universidad Nacional de Córdoba. Investigador Honorario del INICSA (UE CONICET-UNC) e-mail: aeynard@gmail.com

Hasta donde conocemos, poco hay publicado sobre el Profesor Hendrik Weyenbergh, Fundador, primer Decano y primer Profesor dedicación exclusiva de varias asignaturas en la etapa creacional la Facultad de Ciencias Médicas (FCM). Una breve semblanza sobre él publicamos en 2002, en una obra compilada por la Prof. Dra. N. Acerbi Cremades (1) siendo este artículo, al cumplirse ahora 140 años de la fundación de la FCM, una ampliación y actualización de la misma. En el prólogo de su monumental obra "Historia de la Facultad de Ciencias Médicas" que en 1927 publicara la destacada figura del Maestro, Profesor Dr. Felix Garzón Maceda, obra base del presente artículo, él señala "...añorar recuerdos es revivir. Exhibir a la generación usufructuaria o contemporánea y a las generaciones por venir, lo que deben en gratitud y respeto a los que les precedieron es hacer obra útil y de justicia. Los esfuerzos y consagraciones que alentaron los propios tiempos con sus tropiezos, con sus errores y también sus triunfos, dignos son de atención y justiprecio" (2). Se dispone también de una valiosa y completa síntesis de la historia de la FCM de la Prof. Dra. Norma Acerbi Cremades ${ }^{(3)}$.

Advirtiendo que no somos historiadores, es nuestro propósito no solo mencionar, fechas y nombres relacionados a Hendrik Weyenbergh (nótese que su apellido se escribe con " $n$ " y no con " $m$ ", como lo hacemos frecuentemente en Córdoba) sino también de otros precursores, sus colegas de la Academia Nacional de Ciencias y de la UNC, así como políticos, en especial del rector Dr. Manuel L. Lucero, que tuvieron las máximas responsabilidades en la naciente FCM; así mismo, enmarcarlos en las circunstancia que les tocaron vivir en ésta Universidad, en Córdoba, el país y Sudamérica a fines del siglo XIX. Es difícil obtener datos cronológicos precisos de los Maestros cuando de ellos nos alejamos en el tiempo pero más complicado aún es la carencia de información sobre sus vidas, lo que nos desdibuja los seres humanos que fueron, con sus aciertos y errores, sus alegrías y penas. Esta aproximación será opinable, es natural que así sea, pues se basa en la escasa bibliografía disponible, con aspectos subjetivos y pareceres y por algo de tradición oral, que afortunadamente logró transcribirse.
Como se dijo, la obra referente para la historia de la FCM de Córdoba, es sin duda la de Garzón Maceda. Es la primera historia completa de la "Facultad de Ciencias Medicas" de la Universidad Nacional de Córdoba, y aunque luego hubo algunos encomiables aportes $^{(4)}$ hasta la fecha del envío de este articulo al Editor no se ha continuado y completado en alguna publicación, que en su $140^{\circ}$ aniversario parece muy necesaria.

Hendrik Weyenbergh tuvo un papel prominente en la creación de la Facultad redactando su primer plan de estudios. (Figura 1).

Figura 1: Hendryk Weyenbergh en 1876 (es una ampliación de su rostro de Fig.4) y a la derecha, antes de regresar a su patria.
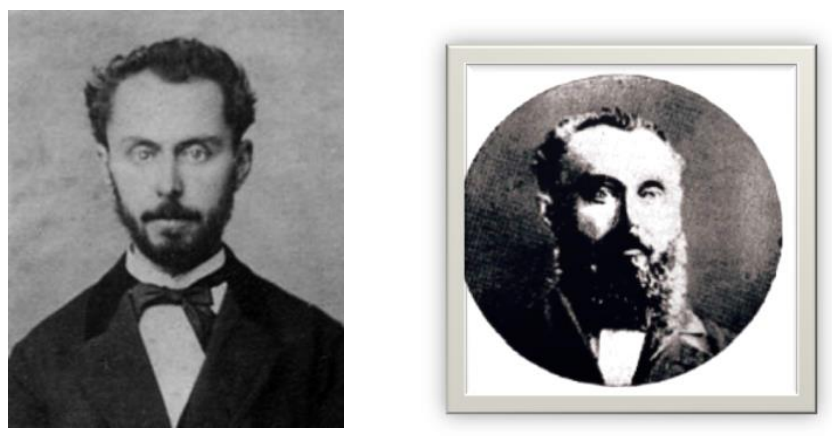

Reconocido por sus publicaciones y el protagonismo que había adquirido en Europa, notable por su juventud y su múltiple condición de médico, zoólogo y naturalista, Weyenbergh fue convocado para dirigir uno de los logros más destacados de Domingo Faustino Sarmiento, como es la Academia Nacional de Ciencias, que fundó en Córdoba.

El Dr. Carlos Hermann Burmeister, ya contratado por Domingo Faustino Sarmiento, distinguía a Weyenbergh entre los científicos europeos de su época y se 10 recomendó al gobierno argentino, que por entonces $y$ muy sabiamente estaba importando científicos para sembrar excelencia científica en las añejas aulas de nuestra Universidad.

Como veremos más adelante, este aluvión de "sabios" provocó sobresaltos y malcontento en la "siesta" social y académica cordobesa, ya señalada con sarcasmo y 
picardía por Sarmiento en su Facundo, en $1845^{(5)}$.

En 1873, residiendo ya en Córdoba y presidiendo la Academia Nacional de Ciencias, Weyenbergh funda el Museo de Zoología de la UNC. El insistía, junto con el médico Manuel M. Espinoza, ante las autoridades Rectorales en la necesidad de la creación de una Facultad de Ciencias Médicas.

Recíprocamente, la misma inquietud ya había nacido y tuvo apoyo inmediato en el entonces Rector de la UNC, el Dr. Manuel Lucero, que transcurría su tercera gestión consecutiva como tal, (Figura 2).

Figura 2: Rector Manuel L. Lucero.,ca 1875.
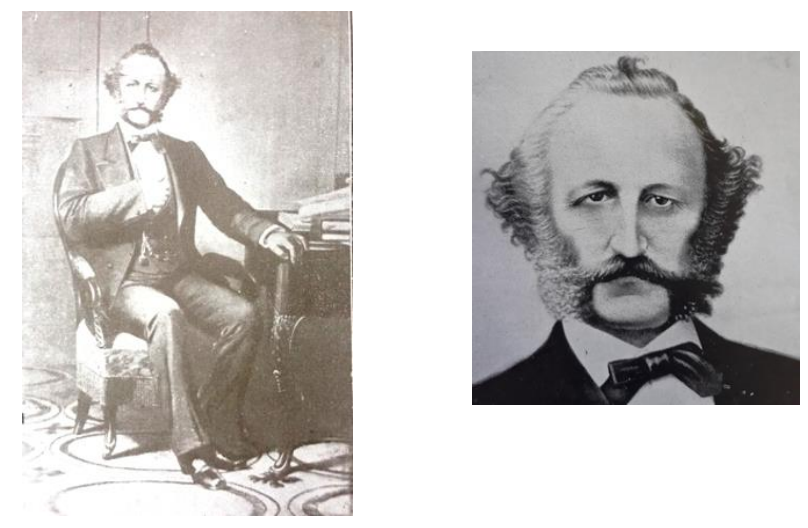

Así, el médico y naturalista Weyenbergh, junto con el Dr. Luis Warcalde, Diputado de la Nación por Córdoba, y el Rector Manuel Lucero conformarán la triada fundacional de nuestra Facultad de Medicina, que nace como tal en 1877, hace 140 años. A esos nombres agregamos el del Gobernador, Dr. Antonio del Viso por el apoyo prestado a ésta arrojada empresa facilitando el acceso al Hospital San Roque para las prácticas de los estudiantes.

Weyenbergh nació en Harlem, Provincia de Noword, Holanda, el 6 de Diciembre de 1842 y habiendo retornado desde Córdoba a Holanda ya enfermo, murió en 1885, a consecuencia de un cáncer con sólo 43 años de edad. Fue sepultado en su tierra natal, en la vecina aldea de Bloemendaal; el nombre de su esposa fue Sjoukje Gorteral.

En 1863, a los 21 años de edad, se graduó de médico en la Universidad de Amsterdam, pero ya antes se había Licenciado en Ciencias Naturales. Hizo su perfeccionamiento científico en Utrecht y en 1872 se doctoró en Zoología en la Universidad de Gôttingen. Había publicado en holandés o alemán, más de 60 monografías científicas y tan tempranamente como 1870 había sido galardonado con una medalla de oro al mérito científico. Entre sus obras más trascendentes se pueden mencionar un libro en 1870, en coautoria con C.K. Hoffmann titulado: 'Die Osteologie und Myologie von Sciurus-vulgaris L., vergliechen mit der Anatomie der Lemuriden und D.", que se puede traducir como "La Osteologia y la Miología del Sciurus-vulgaris $L$. comparado con la Anatomía del Lemuriden and D". Otro de 1872- Beitrage zur Anatomie und Histology der Hemicephalen Dipteren-larven (der Gattung Ctenophora Meig) ó "Contibución a la Anatomía e Histología de la larvas de Hemicephalen Dipteren-larven (Ctenophora Meig) y otro más general, en 1873, titulado " Taak der Dierkunde" ó "Las funciones, o tareas, de la biología Animal". Como puede apreciarse por sus intereses científicos, Weyenbergh era un Zoólogo interesado en la Anatomía Macro y Microscópica (Histología y Embriología) Comparada ${ }^{(2,6)}$.

Weyenbergh llegó a Córdoba en 1872 y fue nombrado Profesor de Zoología y Anatomía Comparada en la Facultad de Ciencias Naturales Físicas y Matemáticas, recientemente creada en la Universidad Nacional de Córdoba. Su aprendizaje del idioma castellano debe haber sido muy veloz, pues en 1873 publicó en español un estudio sobre abejas y apicultura. Durante su estadía en la Argentina publicó cientos de trabajos, principalmente en español, aunque también lo hacía en francés, holandés, latín y alemán. Sus intereses multifacéticos en ciencia se completaban con la publicación de poemas y artículos periodísticos, entre los que se destacaban semblanzas e impresiones de sus vivencias sobre Córdoba y la Argentina de fines del siglo XIX.

El fue, al inicio de la FCM, el Decano, Profesor de Histología Normal y Patológica, Anatomía, Fisiología y único académico, de dedicación exclusiva. Renunció a su estipendio durante bastante tiempo, solicitando por escrito que ese dinero se emplease en la compra de bibliografía y equipamiento para los gabinetes.

Publicó el primer trabajo científico-pedagógico de la Facultad de Medicina de Córdoba, titulado "Principios Histológicos", en 3 tomos. Esta obra fue la principal fuente bibliográfica de los alumnos estudiantes de las primeras promociones hasta que los libros de los Histólogos franceses fueron adquiriéndose de a poco, aunque los alumnos tenían dificultad con el idioma $(2,3,4,7)$. Montó un precario gabinete (laboratorio) formando las primeras colecciones de preparaciones histológicas y anatómicas. A pesar del arduo esfuerzo del Maestro y sus primeros ayudantes, las primeras lecciones eran teóricas, por carencia de microscopios, escasos cadáveres para disección, preparaciones de cera $y$ de otros materiales. La bibliografía que empleaban los docentes de Histología y de patología era escasa, de difícil acceso y los alumnos carecían aun de biblioteca propia de la Facultad. Se estudiaba en los dos volúmenes de los "Manuales" de Weyenbergh y luego los textos de Klein y Variot junto con el Cornil y Ranvier, que eran ediciones de 1885, en francés, idioma que los alumnos no conocían lo suficiente ${ }^{(2,7)}$.

Las circunstancias histórico culturales de América Latina, Argentina y de Córdoba a lo largo de larga lucha académico-política orientada a la creación de la FCM eran muy difíciles. En el Cono Sur hace crisis en 1878 el largo conflicto fronterizo con Chile, instalándose fuerzas chilenas en Santa Cruz, territorio recuperado por la escuadra naval argentina en ríspido episodio ${ }^{(8)}$. Los malones siguen amenazando el Sur de Córdoba y pocos años antes las lanzas de Calfucurá y Namuncurá habían llegado, incluso, hasta La Carlota y vecindades de Rio IV; luego Julio $A$. Roca se hace cargo de la campaña del Desierto cuyos resultados la historia ha juzgado controvertidamente ${ }^{(7-9)}$. Bolivia y 
EDITORIAL

Perú entran en conflicto con Chile por las salitreras, preludio de la Guerra del Pacífico. Ya en 1879, victorioso Roca en la campaña del desierto y con perfil presidencial para sucederlo a Avellaneda, se conforma la "Liga de Gobernadores" con sede en Córdoba, teniendo Roca como contrincante a Bartolomé Mitre y Carlos Tejedor. Buenos Aires era capital de sí misma, de su Provincia y del país y habrá que esperar hasta 1880-y la derrota de Tejedor-, para que Argentina pudiese, por fin, tener su Capital Federal. En Europa se afianza cada vez más la enseñanza laica y común impartida por el Estado y los hermanos países Chile, Perù y Bolivia comienzan la cruenta guerra del Pacífico durante la cual Argentina mantendrá, a duras penas, su neutralidad ${ }^{(7,8)}$

A finales de la década de los '70 e inicios del ' 80 del siglo XIX, el ambiente socio-político de Córdoba estaba muy agitado y el ambiente académico no era ajeno a ello. En efecto, habiendo asumido como Gobernador Antonio del Viso, (originalmente electo como Vice, ante la sorpresiva muerte del Gobernador electo, Clímaco De la Peña) nombra a Miguel Juárez Celman como ministro de Gobierno y simultáneamente se redoblan las reservadas actividades del Club "El Panal", muchos de cuyos muy influyentes miembros, entre ellos su hermano Marcos Juárez, son conocidos masones de Córdoba. Esas reuniones, más o menos encubiertas, tienen como objetivo principal lograr la candidatura a Presidente de la Nación de Julio Argentino Roca y luego, del mismo Miguel Juárez Celman ${ }^{(7,9-12)}$

En ese contexto socio-histórico agitado, cupo principalmente al Dr. Hendrik Weyenbergh y el Dr. Manuel Lucero la concreción de las etapas fundacionales de la FCM. Las figuras de ambos se agigantan cuando se les observa luego de 140 años de aquel trascendental suceso. En tanto que Weyenbergh era hombre de ciencia y gabinete, muy afable con sus alumnos, aparentemente sin mayores relaciones sociales con los cordobeses, Manuel Lucero tenía una presencia muy protagónica y polémica no solo en los Claustros, sino también en la patriarcal y conservadora sociedad de la "Docta".

Manuel Lucio Lucero, hombre de Córdoba (nacido en 1814 en La Población, Departamento San Javier, "Traslasierras") estaba empeñado en la creación de la Facultad de Ciencias Médicas, que al final logró en 1878 siendo Weyenbergh su más entusiasta asociado en la empresa (Figuras 1 y 2). Estudiando lo poco que se conoce de la personalidad de éste quedan algunas preguntas, germen de futuras investigaciones: ¿En qué se basaba ese entendimiento aparentemente tan fluído y franco entre el holandés Weyenbergh y el rector Lucero?.

Este último, según Garzón Maceda, "un hombre detestado por los hombres de escapulario de Córdoba y descripto como masón"(2). Jugando con su nombre, Lucio y Lucero, sumado al hecho de ser soltero, bien plantado (Bello Lucero: "Luzbel"), de verba riquísima, florida, guitarrero y galante, afortunado en lances románticos y libre pensador, se le apodaba también "Lucifer" y "Hereje masón", entre otros motes (2 7,11).
Cauto es Garzon Maceda cuando a él alude diciendo sólo "descripto como masón".

En rigor, el papel de la masonería de Córdoba en los últimos 25 años del siglo XIX, su intrincada $y$ muchas veces, reservada, labor en los hechos políticos y acádemicos en la UNC y en la FCM durante ese período no han sido analizados a fondo aún. Ciertamente, Lucero fue un connotado masón, Venerable Maestro de la Logia Nro. 34 Piedad y Unión de Córdoba, aunque había sido "iniciado" en 1861 en Paraná, en la Logia que fundara Don Justo J. de Urquiza, en 1861.

La masonería, entidad internacionalista por excelencia y placenteramente receptora de calificados extranjeros, era una suerte de Club de destacados cófrates a donde naturalmente acudirán muchos de los "sabios" europeos contratados en la Academia Nacional de Ciencias y Profesores de las nacientes Facultades de Ciencias Exactas Físicas y Naturales y de Ciencias Médicas. ${ }^{(7,11-13)}$, Figuras 3 y 4.

Figura 3: Algunos Miembros de la Academia Nacional de Ciencias de Córdoba, año 1876. Sentados, de izquierda a derecha: Hendryk Weyenbergh (Zoólogo y Médico); Francisco Latzina (Matemático y Geógrafo); Oskar Dôering (Físico). De pié: Georg Hyeronimus (Botánico); Louis Brackebusch (Geólogo); Adolf Dôering (Químico y Geólogo); Afredo Steltzer (Químico)

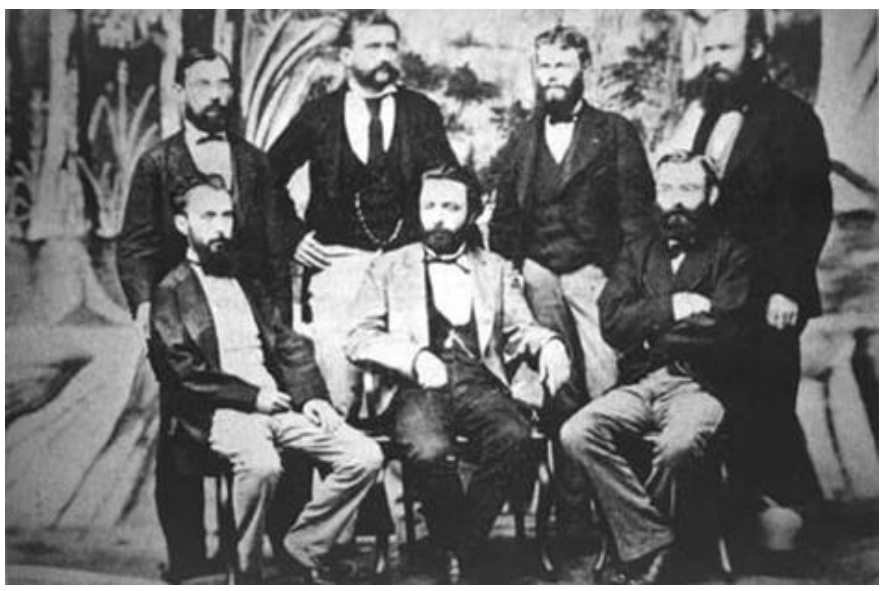

Figura 4: Algunos miembros de la Academia Nacional de Ciencias de Córdoba, año 1876. De izquierda a derecha, de pié: Pablo Lorentz (botánico);Karl Schultze - Sellak; Hendryk Weyenbergh ( Zóologo); sentados: Max Siewert (químico); August Vogler (matemático), Afredo Steltzer.

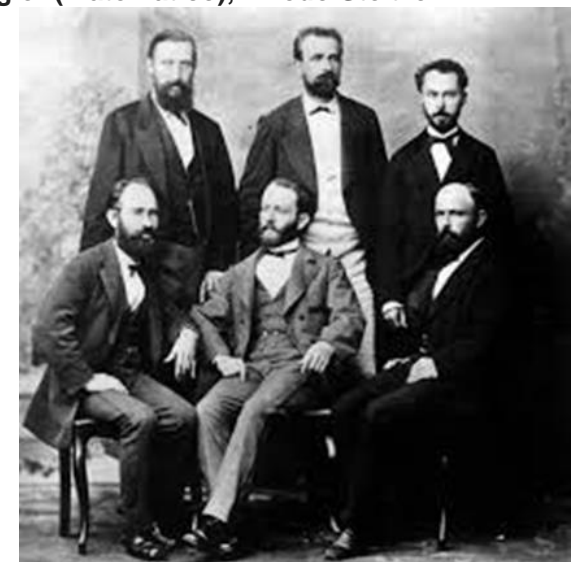

Aunque no hay registros de que Weyenbergh fuera un cófrate de la masonería, sí hizo regalo de una Biblia 
dedicada por él a la Logia Nro. 34, a través de su colega y amigo Oscar Doering, que fue también Venerable Maestro de la misma. Consignan Enrique A. Morra y Alcibíades Lappa, en sus meticulosas investigaciones, que algunos importantes masones de esas Instituciones (sin ser exhaustivos) fueron: German C. Burmeister, Manuel L. Lucero, Adolfo y Oscar Dôering, Federico Schickendantz, Pedro A Conil, Juan Bialet Massé, Pedro Vella, Julio W. Gómez, Carlos Alberto Cassaffousth, José Figueroa Alcorta, Francisco Latzina, Florentino Ameghino, Luis Brackebusch, Eugenio Bachmann, Arturo Von Seelstrang , Luis Vidal Peña, Benjamin Gould, John Macon Thome, Georg Wiggin y Luis Harperah ${ }^{(11-13)}$. La discreción, allá por 1927, de Garzón Maceda, y dado su pertenencia a una tradicional familia, hay que enmarcarla en la siempre presente, y a veces subterránea lucha entre la Córdoba monacal, católica y conservadora frente a la Córdoba libertaria, librepensadora, laica y reformista, especialmente al final del siglo decimonónico ${ }^{(12)}$; Esto podía percibirse hasta entrado el siglo $X X$, según el punto de vista de quien fuera Profesor contratado de Fisiología en la FCM hasta 1926, el alemán Georg Friederich Nicolai, quien Ileno de resentimiento y amargura se despide de la UNC de Córdoba donde ya no se siente útil y es rechazado, con su opúsculo "Homenaje de Despedida a la Tradición de Córdoba Docta y Santa", que por cierto, solo logró imprimirlo en 1927, en Buenos Aires (14). Aunque cargado de subjetividad y negativa emocionalidad vale la pena leerlo y vernos, los cordobeses, reflejados en aquella época en ese espejo tan duro pero no del todo erróneo. Sólo citamos un ejemplo: dice Nicolai... "Y los cordobeses son geniales. Cuando se trataba una vez de proponer una terna para una Cátedra de Zoología, todo el Consejo estaba de acuerdo en que el candidato cordobés no sabía lo más mínimo de la materia, pero estaba de acuerdo también en que esto no importaba y en que él aprendería dando clase..." "(14).

Si así era la Córdoba académica y social aún en los años '20 del siglo pasado habiendo ya acaecido la Reforma del '18, imaginemos a aquellos muchachos, los "sabios" europeos y norteamericanos, en la plenitud de sus jóvenes 35 o 40 años de edad, compartiendo el alemán, inglés o el francés como lenguas comunes, ¿Cómo se divertían, al salir de los silenciosos gabinetes o de las sobrias Aulas del Claustro en la muy recatada ciudad de Córdoba de los '80?.

Enrique A. Morra, citando a Godofredo Lascano Colodrero en su "Retablillo de Córdoba", consigna que estos muchachos se reunían en un "Bar Alemán", entonces en calle San Jerónimo, frente al Banco de la Provincia de Córdoba. Sacudiéndose la solemnidad de su obligada seriedad académica, estos barbudos profesores hacían consumo de abundantísima cerveza y entonces cantaban a voz en cuello canciones de la patria lejana y se comportaban como niños. Espectáculo báquico un poco bizarro era éste para los atildados y solemnes cordobeses observando a estos heréticos con chambergos desquiciados, trajes arrugados, sin ninguna elegancia, riéndose a pulmón lleno. En la madrugada, concluida la juerga, dos o tres coches Mateos depositaban en sus respectivos domicilios a los adormilados doctores $^{(11)}$.

Pero no todo fue grato para Hendryk Weyenbergh. Su vida breve pero intensa tuvo éxitos sí, pero también penas y tristezas. Entre alguna de sus amarguras mencionamos que médicos que él ayudó a instalarse en Córdoba y a conformar el cuerpo académico de la Facultad sospecharon de su capacidad e idoneidad para dictar sus asignaturas; tanto es así, que en 1881 se vio obligado a hacer traducir y legalizar por la Embajada de los Países Bajos, su diploma de médico para mostrárselo a la Academia, o Consejo Directivo de entonces. Pero puntualizó que solo lo hacía por las infamantes dudas surgidas, pues siempre tuvo muy en claro que no deseaba hacer medicina práctica, sino abocarse únicamente a la investigación y a la vida académica. A pesar de tan contundente desmentida, en el "Álbum de la Provincia de Córdoba", edición oficial de 1927, Dutari Rodríguez consigna... "el Dr. Weyembergh no era médico pero fue contratado para dictar la cátedra de Histología que funcionó solamente durante el curso de 1878 con cuarenta alumnos según documentos fehacientes, por más que el Doctor Lastra diga en sus memorias, que hemos visto, que tenía seis."(15). Así, transcurrido pocos años al frente del Decanato, algunos hechos deben haber debilitado su entusiasmo, tal vez su enfermedad, pues queda como opacado por la figura emergente y avasallante del Dr. Luis Rossi, en quien delegará el Decanato en 1881. A pesar de ello, Weyenbergh continuará formando parte de la Academia hasta su regreso definitivo a Holanda en 1884 donde fallece un año después.

Al final de estas reflexiones me imagino, traídos desde el pasado y acompañándolos en una visita por los Hospitales, Laboratorios y Aulas de nuestra FCM, a las venerables figuras del sabio Hendrik Weyenbergh y del Dr. Manuel Lucero. Ahora, transcurrido casi un siglo y medio, complacidos verían ellos a miles de estudiantes no solo de medicina, sino de otras florecientes carreras de las Ciencias de la Salud, provenientes de Córdoba, del país y del extranjero, con sus laboratorios colmados con decenas de laboriosos jóvenes, becarios de investigación y cuyos hospitales, cátedras y servicios cuentan con cientos de entusiastas residentes y sus calificados maestros. $Y$ si alguna carencia se hiciese notar, bienvenidas, pues son las que hay que resolver de ahora en más y sin desmayos por el futuro venturoso de nuestra Facultad de Ciencias Médicas de la Universidad Nacional de Córdoba.

\section{Agradecimientos}

A mi amigo Sr. Wim Huson, de Caastricum, Holanda, quien hizo los contactos con la Biblioteca de Utrecht para ahondar respecto a los datos académicos de H.Weyenbergh. 


\section{EDITORIAL}

\section{Bibliografía}

1. Eynard AR. Historia de la Cátedra de Histología de la Facultad de Ciencias Médicas de la Universidad Nacional de Córdoba y de sus Maestros". En: Libro en conmemoración del 125 Aniversario de la Facultad de Ciencias Médicas, Universidad Nacional de Córdoba, 2002. Editor: Norma Acerbi Cremades, pp 251-260.

2. Garzón Maceda F. Historia de la Facultad de Ciencias Médicas. Publicación Oficial de la Universidad Nacional de Córdoba, Tomo I y II, Imprenta de la Universidad Nacional de Córdoba, Argentina, 1927.

3. Acerbi Cremades, N. Síntesis histórica de la fundación de la Facultad de Ciencias Médicas de Córdoba. En

https://historiamedicacordobesa.blogspot.com.ar /p/historia-de-la-medicina.html. Consultado el 7 Agosto 2017

4. Aznarez, EP. Introducción a la Historia de la Medicina, Revista de la Facultad Ciencias Médicas, 1945, Año III, Nro 4, 95-114.

5. Sarmiento, D.F. Facundo, pp. 142-148, Tomo $X X V$, en colección Grandes Escritores Argentinos, Director Alberto Palcos, WM Jackson, Buenos Aires, Argentina, 1937.

6. Visscher, Harry, Mr , P/a Universiteitsbibliotheek Universiteit Utrecht, PO Box 16007

3500 DA Utrecht, Nederland, e-mail: h.visscher@library.uu.nl. http://www.kb.nl Accedido 8 Septiembre 2001.

7. Bischoff, EU. "Historia de Córdoba", 3a. Ed., Editorial Plus Ultra, Buenos Aires, Argentina, 1977.

8. Paz, RA. El Conflicto pendiente, Fronteras con Chile, Vol 1; El Beagle y el cabo de Hornos, Vol 2. $2 d a$ ed., EUDEBA, Buenos Aires, Argentina, 1981.

9. Zeballos, ES. Callvucurá y la dinastía de los Piedras. Vol 1 y 2, Centro Editor de América Latina, Buenos Aires, Argentina, 1981.

10. Historia de la Literatura Argentina, del Romanticismo al Naturalismo, Acontecimientos históricos y Culturales 1853-1900, vol 2. Centro Editor de América Latina, Buenos Aires, Argentina, 1980

11. Morra, EA. Historia de la Masonería en Córdoba, vol 1, 1aㅡ Ed. del Autor, ISBN 978-98733-0426-2, Centro Editor de Córdoba, Córdoba, Argentina, 2011.

12. Corbière EJ. La Masonería II. Tradición y Revolución. Editorial Sudamericana, Buenos Aires, Argentina, ISBN 950-07-1953-3, 2001.

13. Lappas, A. La Masonería argentina a través de sus hombres, Buenos Aires, Argentina, Edición del autor, 2da. Ed., 1966

14. Nicolai, GF. Homenaje de despedida a la Tradición de Córdoba Docta y Santa, 1a Edición : Sociedad de Publicaciones El Inca, Diciembre de 1927, Reimpresión facsimilar por
Ed. Universidad Nacional de Córdoba, ISBN 978-950-33-0678-9, 2008

15. Escobar Uribe A, Ellauri Obligado, G . "Álbum de la Provincia de Córdoba 1927". Talleres Gráficos La Elzeviriana", Córdoba, 1927. 